\title{
Review of: "A scanning dynamic collimator for spot- scanning proton minibeam production"
}

Toshiyuki Toshito

Potential competing interests: The author(s) declared that no potential competing interests exist.

This study proposes new irradiation method to deliver minibeam for proton therapy using a dynamic collimator system with single slit instead of using a static multi-slit collimator which have been used for previous studies. Because of very attractive biological benefit of minibeam reported in preclinical studies, early realization of proton therapy using minibeam is desired. Proposed concept is very flexible, it has great potential to expand the possibility of minibeam irradiation especially for real-time online adaptive therapy.

Observed unequal peak for dynamic 5-slit minibeam array was attributed to a misalignment. Required mechanical precision and the effect of beam profile of scanning beam should be considered more. I think that iron is better material for collimator than brass due to its mechanical precision and durability. My major concern is if the proposed design of two-plane dynamic collimator on a hexapod works well with desired precision. Also, degradation of throughput will be inevitable. I hope these issues will be overcome in the future. 